\title{
OPINION
}

\section{The BAFFling effects of rituximab in lupus: danger ahead?}

Michael R. Ehrenstein and Charlotte Wing

\begin{abstract}
Sub-optimal trial design and concurrent therapies are thought to account for the unexpected failure of two rituximab clinical trials in patients with systemic lupus erythematosus (SLE). However, in this Perspective we propose an alternative explanation: that rituximab can trigger a sequence of events that exacerbates disease in some lupus patients. Post-rituximab SLE flares that are characterized by high anti-dsDNA antibodies are associated with elevated circulating BAFF (B-cell-activating factor), and a high proportion of plasmablasts within the B cell pool. BAFF not only perpetuates autoreactive B cells (including plasmablasts), particularly when B-cell numbers are low, but also stimulates $\mathrm{T}$ follicular helper $\left(\mathrm{T}_{\mathrm{FH}}\right)$ cells. Moreover, plasmablasts and $\mathrm{T}_{\mathrm{FH}}$ cells promote each others' formation. Thus, repeated rituximab infusions can result in a feedback loop characterized by ever-rising BAFF levels, surges in autoantibody production and worsening of disease. We argue that B-cell depletion should be swiftly followed by BAFF inhibition in patients with SLE.
\end{abstract}

\section{Competing interests}

Professor Ehrenstein is Chief Investigator for BEAT-LUPUS, a trial combining rituximab and belimumab for patients with SLE funded by Arthritis Research UK, UCLH Biomedical Research Centre and GSK.

Systemic lupus erythematosus (SLE) is a chronic multisystem autoimmune disease associated with substantial morbidity and some mortality ${ }^{1}$ A considerable body of evidence implicates B cells in the pathogenesis of SLE, and successful use of the B-cell-depleting agent rituximab in patients with SLE has been reported in numerous open-label studies (reviewed elsewhere) ${ }^{2}$. However, two randomized controlled trials of rituximab therapy in patients with SLE did not meet their primary end points: EXPLORER ${ }^{3,4}$ and LUNAR $^{5}$. It has been cogently argued that various aspects of trial design could account for these failures ${ }^{2}$, and accordingly further trials of rituximab in patients with SLE are now planned or underway. However in this Perspective, we propose a different explanation for the failure of these trials: specifically that rituximab therapy triggers a surge in BAFF (B-cell-activating factor, also known as TNF ligand superfamily member 13B or BLyS) which exacerbates disease in some patients with SLE. 


\section{Plasmablasts and SLE flare}

Patients with SLE respond differentially to rituximab according to their anti-dsDNA antibody levels, the duration of B-cell depletion, and the type of B cell that dominates the early repopulation phase. Disease returns most quickly in patients whose B cells repopulate rapidly ${ }^{6}$. In this section, we discuss the evidence that an increased frequency of circulating plasmablasts found in the B-cell pool following rituximab therapy is associated with lupus flares characterised by high anti-DNA antibody levels.

Among patients with SLE who relapse after rituximab therapy, those with high antidsDNA antibody levels have significantly fewer B-cells at the time of relapse compared to those who flare without increased anti-DNA antibodies ${ }^{6}$. Post-rituximab flare in patients with high anti-dsDNA antibodies was characterized by an increased percentage of circulating plasmablasts within the repopulating B cell pool, compared to patients who remained in remission and those who relapsed without increased anti-DNA antibodies ${ }^{6}$. Elevated plasmablast numbers following rituximab treatment has been associated with a shorter interval to subsequent relapse ${ }^{7}$. An increased frequency of peripheral blood plasmablasts is also associated with active disease in patients with SLE, and this B-cell subset is considered to have a role in driving disease ${ }^{8}$. The number of $\mathrm{CD} 27^{\text {high }}$ plasmablasts in patients with SLE is strongly correlated with SLE disease activity indices, and with anti-dsDNA antibodies ${ }^{9}$. Despite low overall B-cell numbers following B-cell depletion therapy, a high percentage of plasmablasts, presumably producing autoantibodies, within the residual B-cell pool could trigger an SLE flare. Patients who have a high proportion of plasmablasts also often have few transitional B cells, a compartment rich in regulatory B cells. Deficiency in this suppressive population could contribute to an earlier relapse in disease ${ }^{10}$.

Human peripheral blood plasmablasts promote the differentiation of $\mathrm{T}$ follicular helper $\left(\mathrm{T}_{\mathrm{FH}}\right)$ cells, partly via IL-6 $6^{11,12}$. $\mathrm{T}_{\mathrm{FH}}$ cells can in turn stimulate plasmablast formation, thereby creating a positive feedback loop and perpetuating disease (Fig. 1). In support of these observations in humans, differentiation of $\mathrm{T}_{\mathrm{FH}}$ cells in mouse models of infection is also dependent upon B-cell production of IL- $6^{13}$. The frequency of $\mathrm{T}_{\mathrm{FH}}$ cells correlates with plasmablast numbers, anti-dsDNA antibody levels and disease activity (particularly the severity of glomerulonephritis) in SLE ${ }^{14-16}$. Moreover, circulating $\mathrm{T}_{\mathrm{FH}}$ cells in patients with SLE are capable of driving differentiation of $\mathrm{B}$ cells into plasmablasts in vitro ${ }^{17}$. Ectopic clusters of $\mathrm{T}_{\mathrm{FH}}$ cells and $\mathrm{B}$ cells are present in the inflamed kidneys of patients with SLE and, 
together with conventional germinal centre formation, probably contribute to the pathogenesis of this disease ${ }^{18}$. Evidence from animal models of lupus suggests that abnormal germinal centre activity and $\mathrm{T}_{\mathrm{FH}}$ cells orchestrate the generation of lupus-related autoantibodies. Mice homozygous for $R c 3 h 1^{\text {san }}$ (the san allele encoding the sanroque form of roquin-1, a RING-type ubiquitin ligase) develop germinal centres, excessive $\mathrm{T}_{\mathrm{FH}}$ cell numbers and features of lupus such as glomerulonephritis ${ }^{19}$. Adoptive transfer of sanroqueexpressing $\mathrm{T}_{\mathrm{FH}}$ cells into wild-type recipients resulted in spontaneous germinal centre formation, underscoring the direct role of $\mathrm{T}_{\mathrm{FH}}$ cells in lupus-associated autoimmunity. In another lupus model, BXD2 mice, $\mathrm{T}_{\mathrm{FH}}$ cells drive the increased autoantibody levels and enhanced germinal centre B-cell responses through an IL-21 dependent mechanism ${ }^{20}$.

\section{Rituximab disturbs BAFF homeostasis}

The predominance of plasmablasts in the repopulated B cells (in the context of overall B-cell lymphopenia) and the potential of plasmablasts to drive $\mathrm{T}_{\mathrm{FH}}$ cell formation might encourage clinicians to consider the use of increasing doses of rituximab, as has been suggested for rheumatoid arthritis (RA) ${ }^{21}$. However, post-rituximab SLE flares that are accompanied by elevated anti-dsDNA antibody levels and low B-cell numbers are closely associated with increased BAFF levels ${ }^{22}$. BAFF is an important physiological mediator of B-cell homeostasis, and has a role in regulating survival and self-tolerance during B-cell development ${ }^{23}$. Serum BAFF is elevated immediately after rituximab therapy in patients with SLE, but initial reports indicated that BAFF levels reverted to pretreatment values by 6 months, and was not associated with clinical relapse ${ }^{24}$. Similar observations were made in patients with RA treated with rituximab, in whom BAFF levels normalized upon B-cell repopulation and did not correlate with disease relapse ${ }^{25}$. Although these early data did not suggest that elevated BAFF levels directly contributed to disease flare post-rituximab the repopulated B-cells could be initially skewed towards an autoreactive repertoire ${ }^{23}$. Whether a transient elevation in serum BAFF that returned to baseline prior to a relapse in disease would have an enduring adverse impact upon the B-cell repertoire remained uncertain. However, a subsequent study clearly showed that elevated BAFF levels can persist in patients with SLE but only in those who relapse with high anti-dsDNA antibody levels after rituximab treatment ${ }^{22}$, and that these high BAFF levels are likely to provide an environment in which autoreactive B cells flourish. By contrast, patients who flare following B-cell depletion but do not display high anti-dsDNA antibody levels do not have elevated serum BAFF.

Studies in transgenic mice suggest that survival of autoreactive B-cell clones requires 
higher levels of BAFF than does maintenance of the self-tolerant population ${ }^{26}$. Consequently, elevated BAFF lowers the stringency of B-cell selection and thereby rescues autoreactive clones that would otherwise be deleted ${ }^{27-29}$. In addition, BAFF supports the survival of plasmablasts, ${ }^{30}$ whereas BAFF inhibition reduces the differentiation of naive marginal zone $\mathrm{B}$ cells into plasmablasts ${ }^{31}$. Increased production of BAFF by dendritic cells promotes plasmablast formation in patients with $\mathrm{SLE}^{32}$. Rising levels of BAFF following rituximab therapy, coupled with reduced B-cell competition, could remove the brakes on autoreactive B cells (including plasmablasts) and thereby trigger an SLE flare (Fig. 2).

BAFF was first linked to lupus-like autoimmunity following the finding that mice engineered to constitutively express BAFF develop a lupus-like syndrome associated with autoantibodies against nuclear antigens ${ }^{33,34}$. Serum BAFF is elevated in lupus-prone NZB/W F1 mice and treatment of these mice with a BAFF antagonist ameliorated the progression of disease and improved survival ${ }^{35}$. Clinical studies demonstrate that BAFF is overexpressed, either intermittently or persistently, in many patients with $\mathrm{SLE}^{36,37}$, and that levels of BAFF correlate with serological and clinical indices of disease activity in such patients ${ }^{38,39}$. Myeloid cells, B and T cells, and neutrophils are all thought to contribute to the BAFF excess observed in SLE, which is induced by a variety of signals (including type I interferons ${ }^{40-42}$ and TLR9 activation ${ }^{43}$ ). In addition, the B-cell lymphopenia associated with active SLE $^{8}$ could also trigger a compensatory release of BAFF. Belimumab, a BAFF-neutralizing monoclonal antibody, has shown modest efficacy in phase 3 clinical trials, in patients with joint and skin manifestations of SLE, validating BAFF as an important novel therapeutic target $^{44}$. Another therapeutic reagent that targets BAFF, tabalumab, has also shown some promise in phase 3 clinical trials, although a number of key end points were not achieved ${ }^{45,46}$.

Some debate remains as to which of the interactions between BAFF and its three receptors holds the key to pathogenicity in SLE, and it is possible that they all contribute. Knockout studies in the NZM 2328 lupus-prone mouse have demonstrated that any single BAFF receptor is dispensable to the development of lupus. ${ }^{47}$ In vitro, BAFF has a proliferative effect on human mesangial cells via a BAFF-BAFF-R (also known as TNF receptor superfamily member $13 \mathrm{C}$ ) interaction on the cell membrane ${ }^{48}$. In BAFF transgenic mice, deletion of TACI (also known as TNF receptor superfamily member 13B) on B cells protects against BAFF-mediated autoimmunity ${ }^{49}$. The least well-studied BAFF receptor (BCMA, also known as TNF receptor superfamily member 17) has a role in maintaining the survival of plasma cells,${ }^{50}$ but its exact role in autoimmunity remains unclear ${ }^{51,52}$. The generation of two different BCMA-deficient lupus-prone mouse models, B6.lpr Tnfrsf $17^{-1-}$ 
and Nba2 Tnfrsf $17^{-1-}$ mice, which were expected to display milder disease than their wildtype counterparts, in fact developed accelerated proliferation of B cells and plasma cells with increased autoantibody production and early lethality ${ }^{53}$. These observations suggest that in the context of autoimmunity, BCMA might in fact have an inhibitory role in B-cell activation $^{53}$. However, BCMA deficiency in an alternative mouse model of lupus (NZM 2328) resulted in similar morbidity to that observed in the wild-type NZM 2328 mouse $^{47}$. Interestingly, subsequent experiments in these mice with varying pairs of BAFF receptor deletions resulted in different clinical courses of disease: mice with BAFF-R and TACI deletions, or BAFF-R and BCMA deletions, developed attenuated disease ${ }^{54}$.

BAFF is also involved in regulation of $\mathrm{T}_{\mathrm{FH}}$ cells (Fig. 1). BAFF directly stimulates $\mathrm{T}$ cell proliferation and cytokine production ${ }^{55}$, 56; moreover, BAFF blockade in BCMAdeficient, autoimmune disease-prone mice resulted in markedly reduced numbers of $\mathrm{T}_{\mathrm{FH}}$ cells (as well as reductions in germinal centre B cells and plasma cells) in the spleens of these mice, suggesting an important role for BAFF in the maintenance of $\mathrm{T}_{\mathrm{FH}}$ cells in the germinal centre $^{57}$. However, administration of recombinant BAFF to BCMA-deficient mice (but not to wild-type control mice) increased the numbers of total and germinal centre $\mathrm{T}_{\mathrm{FH}}$ cells, germinal centre B cells and plasma cells. These findings suggest that in the absence of BCMA, excessive BAFF signalling via its two other receptors (BAFF-R, TACI, or both) could be responsible for the proliferation of $\mathrm{T}_{\mathrm{FH}}$ cells, germinal centre $\mathrm{B}$ cells and splenic plasma cells.

Analysis of human $\mathrm{T}_{\mathrm{FH}}$ cells from patients with SLE did not reveal any evidence of BCMA expression; however, the percentage of $\mathrm{T}_{\mathrm{FH}}$ cells that expressed BAFF-R was increased in $\mathrm{T}_{\mathrm{FH}}$ cells from patients with SLE compared with those from healthy donors ${ }^{57}$. We might speculate, therefore, that signals transduced through BAFF-R on human $\mathrm{T}_{\mathrm{FH}}$ cells could lead to uncontrolled $\mathrm{T}_{\mathrm{FH}}$ cell differentiation and accumulation, whereas (at least in mice) $\mathrm{BCMA}$ has a role in downregulating $\mathrm{T}_{\mathrm{FH}}$. Local $\mathrm{BAFF}$ production by $\mathrm{T}_{\mathrm{FH}}$ cells has been identified as an important factor promoting the survival of high-affinity B cells in the germinal centre ${ }^{58}$. If this same mechanism is present in an autoreactive setting, it could foster diseases such as $\mathrm{SLE}^{59}$. Thus, development of therapies that target BAFF receptors might be an alternative strategy to BAFF blockade in SLE, but careful analysis of its effects in both mice and humans will be required if untoward effects are to be avoided.

These various lines of evidence suggest, therefore, that B-cell depletion further distorts BAFF homeostasis in patients with SLE, leading to a closer association between BAFF levels and disease exacerbation than is evident in patients with SLE who have not been 
treated with rituximab. This situation might arise because the loss of B cells following rituximab therapy largely removes the major 'users' of circulating BAFF, thereby raising serum BAFF levels ${ }^{60}$. This mechanism, as well as the increased production of BAFF that drives B-cell repopulation, could contribute to the increased levels of BAFF observed in patients who relapse after rituximab therapy compared to both those in remission and those who experience flare prior to B-cell depletion ${ }^{22}$ (Fig. 2).

Changes in serum BAFF levels also correlate positively with changes in anti-dsDNA antibody levels during relapse or remission after rituximab treatment: higher BAFF levels during flare were associated with higher anti-dsDNA levels ${ }^{22}$. Of greater concern is that consecutive flares in a proportion of previously rituximab-treated patients were characterized by incremental increases in both anti-dsDNA antibody and BAFF levels following each cycle of rituximab ${ }^{22}$, (Fig. 3). Indeed, approximately half of patients who relapse following rituximab therapy show increasing levels of BAFF and a rise in anti-dsDNA antibody levels ${ }^{22}$. These effects appear to be most marked in a subset of patients who undergo multiple cycles of rituximab therapy ${ }^{22}$, highlighting a potential danger of successive rituximab treatments for some patients, especially as the most recent analysis of the efficacy of repeated infusions of rituximab revealed that one-third of the patients studied underwent three or more cycles of B-cell-depletion therapy ${ }^{61}$. We envisage the existence of a BAFF-driven feedback loop between plasmablasts and $\mathrm{T}_{\mathrm{FH}}$ cells, gaining ever-increasing momentum with successive rituximab infusions, and leading to worsening disease and accumulating organ damage. This may only apply to patients whose post-rituximab flare is associated with anti-DNA antibodies. Further work is needed to establish the effects of repeated rituximab infusions in patients with SLE, and to elucidate the mechanisms controlling BAFF levels, B-cell repopulation and disease relapse.

A comparison of the repeated use of B-cell depleting therapy in SLE and in RA (in which rituximab has proven efficacy) reveals differences with respect to BAFF levels after rituximab treatment. Firstly, although BAFF levels are elevated in rituximab-treated patients with RA, each successive flare does not lead to further incremental increases in BAFF levels at onset of the next relapse ${ }^{62}$. The authors concluded that elevated serum BAFF levels were not related to the presence of absence of active disease ${ }^{62}$. These differences might contribute to why rituximab's effectiveness, including in patients receiving repeated courses of treatment, has been demonstrated in RA but not in SLE.

\section{The case for sequential therapy}


The principle of targeting more than one pathogenetic mechanism to increase the efficacy of targeted therapies is gaining traction in several autoimmune disease settings. For example, multiple pathways could be targeted sequentially, to first induce remission and then to restore a state of immune tolerance to prevent reactivation of the disease process ${ }^{63}$. A thorough understanding of the mechanism of action of each biological therapy is important in deciding which combinations will work best to prevent resurgence of disease.

The above evidence suggests that the increasing BAFF levels that can arise following repeated cycles of rituximab have the potential to create more aggressive disease and consequent organ damage for some patients with SLE. Thus, rituximab therapy might temporarily improve the disease but its repeated use could worsen patients' long term outcomes. An obvious solution would be to combine rituximab with BAFF blockade, an approach that has been previously proposed to achieve sustained remission in patients with $\mathrm{SLE}^{60}$. In mice, a combination of B-cell depletion and BAFF inhibition removes B cells in the marginal zone and follicular compartments more effectively than either treatment alone ${ }^{64}$. Also in mice, a combination of B-cell depletion therapy and BAFF blockade was superior to B-cell depletion alone with respect to reducing the numbers of plasmablasts and plasma cells, as well as reducing disease severity, in three different models of lupus ${ }^{65}$.

Belimumab has been used prior to rituximab treatment in patients with chronic lymphoid leukaemia. In this disease, the ability of natural killer (NK) cells to directly lyse malignant cells and to mediate antibody-dependent cell-mediated cytotoxicity is compromised. NK cells produce BAFF, particularly when activated, and this increase in BAFF enhances the metabolic activity of malignant cells and reduces their susceptibility to both direct NK-cell lysis and rituximab-induced antibody-dependent cell-mediated cytotoxicity. ${ }^{66}$ Pretreatment with belimumab restored the sensitivity of malignant cells to rituximab-induced NK cell lysis.

We propose that post-rituximab flares characterized by increasing BAFF levels, low B-cell numbers and high anti-dsDNA antibodies could be effectively addressed by administration of a BAFF-blocking agent immediately following B-cell depletion. Two trials of rituximab followed by belimumab therapy are currently recruiting: the open-label CALIBRATE study (Rituximab and Belimumab for Lupus Nephritis) ${ }^{67}$ and the SYNBIoSe study (Synergetic B-Cell Immodulation in SLE) ${ }^{68}$. A third randomized and double-blinded trial (unlike the other two which are open label) comparing belimumab with standard care in patients after rituximab therapy (BEAT-LUPUS) will soon be recruiting in the UK. In addition to determining the efficacy of sequential B-cell-depleting and anti-BAFF therapy, 
these studies will provide a useful opportunity to dissect B-cell kinetics in rituximab-treated patients. Comparing the phenotype, clonal relationships, and the associated autoreactivity of B cells present at relapse and remission in rituximab-treated patients with SLE, with or without the addition of belimumab, will yield insight into the mechanisms of relapse and whether tolerance can be restored. As an alternative to belimumab, conventional immunosuppressive agents (such as mycophenolate mofetil) could potentially be administered to suppress the surge in BAFF levels post-rituximab, although our observations of incremental rises in BAFF levels usually occur on the background of either mycophenolate or azathioprine therapy.

Although belimumab and rituximab were administered together in preclinical studies in mice ${ }^{64,65}$, it is unclear whether simultaneous administration is required or indeed the safest approach. The duration and magnitude of B-cell depletion induced by simultaneous administration of combinations of belimumab and rituximab is unknown in patients. BAFF levels rise soon after rituximab therapy but it is very unusual for patients to experience an SLE flare before their B cell levels have risen to $>0.01 \times 10^{9}$ cells $/ 1$, or before 2 months after rituximab therapy ${ }^{6}$. We would accordingly recommend a flare-prevention strategy based on administration of belimumab 2 weeks after the final rituximab infusion (although we recognize that these timings might need to be revised). Such sequential administration might prevent the surge in BAFF levels from altering the B-cell autoreactive repertoire. Whether this approach would increase the patient's risk of infection is also unknown, although no safety signal was seen in mouse studies using combination B cell depletion and BAFF inhibition $^{64,65}$. It would be important to monitor B-cell repopulation and immunoglobulin levels in patients receiving belimumab after rituximab as surrogate indicators of safety with respect to infection. Moreover, patient's responses to vaccination during and after this combination treatment are likely to be limited, and patients should be advised to receive relevant vaccines some time before rituximab and belimumab are administered.

\section{Conclusions}

Successive disease flares after rituximab therapy in patients with SLE can be marked by rising levels of anti-dsDNA antibodies, a high frequency of plasmablasts, and mounting levels of BAFF. Sequential treatments with rituximab appear to cause these incremental increases in BAFF levels - which, in the setting of low B-cell numbers, enable autoreactive $\mathrm{B}$ cells to thrive. BAFF can also promote the formation of plasmablasts and $\mathrm{T}_{\mathrm{FH}}$ cells and, therefore, accelerate germinal centre activity, leading to the expansion of autoantibody- 
secreting plasma cells. Compounding this effect, the early increase in plasmablast numbers after B-cell-depletion therapy enhances the formation of $\mathrm{T}_{\mathrm{FH}}$ cells, creating a positive feedback loop that perpetuates antibody-driven inflammation in SLE. This sequence of events could explain why some patients with SLE fail to respond (or become refractory) to B-cell depletion therapy.

The evidence presented above provides the rationale for the addition of BAFF inhibition to prevent flares in rituximab-treated lupus patients. Encouraging results have been obtained using this approach in animal models, and randomized controlled trials in humans are underway or about to start. If successful, this new treatment paradigm might prove a major leap forward in controlling SLE and inducing sustained remission in these patients.

Centre for Rheumatology, Division of Medicine, University College London, 5 University Street, London, WC1E 6JF, UK (M.R.E., C.W.).

\section{Correspondence to: M.R.E.}

m.ehrenstein@ucl.ac.uk

\section{References}

1. Thomas, G. et al. Mortality associated with systemic lupus erythematosus in France assessed by multiple cause-of-death analysis: The MORTALUP Study. Arthritis Rheumatol (2014).

2. Furtado, J. \& Isenberg, D.A. B cell elimination in systemic lupus erythematosus. Clin Immunol 146, 90-103 (2013).

3. Merrill, J.T. et al. Efficacy and safety of rituximab in moderately-to-severely active systemic lupus erythematosus: the randomized, double-blind, phase II/III systemic lupus erythematosus evaluation of rituximab trial. Arthritis Rheum 62, 222-33 (2010).

4. Merrill, J. et al. Assessment of flares in lupus patients enrolled in a phase II/III study of rituximab (EXPLORER). Lupus 20, 709-16 (2011).

5. Rovin, B.H. et al. Efficacy and safety of rituximab in patients with active proliferative lupus nephritis: the Lupus Nephritis Assessment with Rituximab study. Arthritis Rheum 64, 1215-26 (2012).

6. $\quad$ Lazarus, M.N., Turner-Stokes, T., Chavele, K.M., Isenberg, D.A. \& Ehrenstein, M.R. B-cell numbers and phenotype at clinical relapse following rituximab therapy differ in SLE patients according to anti-dsDNA antibody levels. Rheumatology (Oxford) 51, 1208-15 (2012).

7. Vital, E.M. et al. B cell biomarkers of rituximab responses in systemic lupus erythematosus. Arthritis Rheum 63, 3038-47 (2011).

8. Odendahl, M. et al. Disturbed peripheral B lymphocyte homeostasis in systemic lupus erythematosus. J Immunol 165, 5970-9 (2000).

9. Jacobi, A.M. et al. Correlation between circulating CD27high plasma cells and disease activity in patients with systemic lupus erythematosus. Arthritis Rheum 48, 1332-42 (2003).

10. Bosma, A., Abdel-Gadir, A., Isenberg, D.A., Jury, E.C. \& Mauri, C. Lipid-antigen presentation by CD1d(+) B cells is essential for the maintenance of invariant natural killer T cells. Immunity 36, 477-90 (2012). 
11. Chavele, K.M., Merry, E. \& Ehrenstein, M.R. Cutting edge: circulating plasmablasts induce the differentiation of human T follicular helper cells via IL-6 production. $J$ Immunol 194, 2482-5 (2015).

12. de Wit, J. et al. Human B cells promote T-cell plasticity to optimize antibody response by inducing coexpression of $\mathrm{T}(\mathrm{H}) 1 / \mathrm{T}(\mathrm{FH})$ signatures. J Allergy Clin Immunol 135, 1053-60 (2015). 13. Karnowski, A. et al. B and T cells collaborate in antiviral responses via IL-6, IL-21, and transcriptional activator and coactivator, Oct2 and OBF-1. J Exp Med 209, 2049-64 (2012).

14. Feng, X. et al. Inhibition of aberrant circulating Tfh cell proportions by corticosteroids in patients with systemic lupus erythematosus. PLoS One 7, e51982 (2012).

15. Simpson, N. et al. Expansion of circulating $\mathrm{T}$ cells resembling follicular helper $\mathrm{T}$ cells is a fixed phenotype that identifies a subset of severe systemic lupus erythematosus. Arthritis Rheum $\mathbf{6 2}$, 234-44 (2010).

16. Choi, J.Y. et al. Circulating follicular helper-like T cells in systemic lupus erythematosus: association with disease activity. Arthritis Rheumatol 67, 988-99 (2015).

17. Zhang, X. et al. Circulating CXCR5+CD4+helper T cells in systemic lupus erythematosus patients share phenotypic properties with germinal center follicular helper $\mathrm{T}$ cells and promote antibody production. Lupus (2015).

18. Liarski, V.M. et al. Cell distance mapping identifies functional $\mathrm{T}$ follicular helper cells in inflamed human renal tissue. Sci Transl Med 6, 230ra46 (2014).

19. Linterman, M.A. et al. Follicular helper T cells are required for systemic autoimmunity. $J$ Exp Med 206, 561-76 (2009).

20. Kim, Y.U., Lim, H., Jung, H.E., Wetsel, R.A. \& Chung, Y. Regulation of autoimmune germinal center reactions in lupus-prone BXD2 mice by follicular helper T cells. PLoS One 10, e0120294 (2015).

21. Vital, E.M., Dass, S., Buch, M.H., Rawstron, A.C. \& Emery, P. An extra dose of rituximab improves clinical response in rheumatoid arthritis patients with initial incomplete B cell depletion: a randomised controlled trial. Ann Rheum Dis 74, 1195-201 (2015).

22. Carter, L.M., Isenberg, D.A. \& Ehrenstein, M.R. Elevated serum BAFF levels are associated with rising anti-double-stranded DNA antibody levels and disease flare following B cell depletion therapy in systemic lupus erythematosus. Arthritis Rheum 65, 2672-9 (2013).

23. Cancro, M.P., D'Cruz, D.P. \& Khamashta, M.A. The role of B lymphocyte stimulator (BLyS) in systemic lupus erythematosus. J Clin Invest 119, 1066-73 (2009).

24. Cambridge, G. et al. B cell depletion therapy in systemic lupus erythematosus: relationships among serum B lymphocyte stimulator levels, autoantibody profile and clinical response. Ann Rheum Dis 67, 1011-6 (2008).

25. Cambridge, G. et al. Circulating levels of B lymphocyte stimulator in patients with rheumatoid arthritis following rituximab treatment: relationships with B cell depletion, circulating antibodies, and clinical relapse. Arthritis Rheum 54, 723-32 (2006).

26. Thien, M. et al. Excess BAFF rescues self-reactive B cells from peripheral deletion and allows them to enter forbidden follicular and marginal zone niches. Immunity 20, 785-98 (2004).

27. Lesley, R. et al. Reduced competitiveness of autoantigen-engaged B cells due to increased dependence on BAFF. Immunity 20, 441-53 (2004).

28. Thorn, M., Lewis, R.H., Mumbey-Wafula, A., Kantrowitz, S. \& Spatz, L.A. BAFF overexpression promotes anti-dsDNA B-cell maturation and antibody secretion. Cell Immunol 261, 9$22(2010)$.

29. Liu, Z. \& Davidson, A. BAFF and selection of autoreactive B cells. Trends Immunol 32, 38894 (2011).

30. Avery, D.T. et al. BAFF selectively enhances the survival of plasmablasts generated from human memory B cells. J Clin Invest 112, 286-97 (2003).

31. Balázs, M., Martin, F., Zhou, T. \& Kearney, J. Blood dendritic cells interact with splenic marginal zone B cells to initiate T-independent immune responses. Immunity 17, 341-52 (2002).

32. Joo, H. et al. Serum from patients with SLE instructs monocytes to promote IgG and IgA plasmablast differentiation. J Exp Med 209, 1335-48 (2012).

33. Mackay, F. et al. Mice transgenic for BAFF develop lymphocytic disorders along with autoimmune manifestations. J Exp Med 190, 1697-710 (1999). 
34. Khare, S.D. et al. Severe B cell hyperplasia and autoimmune disease in TALL-1 transgenic mice. Proc Natl Acad Sci U S A 97, 3370-5 (2000).

35. Gross, J.A. et al. TACI and BCMA are receptors for a TNF homologue implicated in B-cell autoimmune disease. Nature 404, 995-9 (2000).

36. Cheema, G.S., Roschke, V., Hilbert, D.M. \& Stohl, W. Elevated serum B lymphocyte stimulator levels in patients with systemic immune-based rheumatic diseases. Arthritis Rheum 44, 1313-9 (2001).

37. Stohl, W. et al. B lymphocyte stimulator overexpression in patients with systemic lupus erythematosus: longitudinal observations. Arthritis Rheum 48, 3475-86 (2003).

38. Petri, M. et al. Association of plasma B lymphocyte stimulator levels and disease activity in systemic lupus erythematosus. Arthritis Rheum 58, 2453-9 (2008).

39. Zhao, L.D. et al. Expressions of BAFF/BAFF receptors and their correlation with disease activity in Chinese SLE patients. Lupus 19, 1534-49 (2010).

40. Lopez, P. et al. Interferon-alpha-induced B-lymphocyte stimulator expression and mobilization in healthy and systemic lupus erthymatosus monocytes. Rheumatology (Oxford) 53, 2249-58 (2014).

41. Panchanathan, R. \& Choubey, D. Murine BAFF expression is up-regulated by estrogen and interferons: implications for sex bias in the development of autoimmunity. Mol Immunol 53, 15-23 (2013).

42. Litinskiy, M.B. et al. DCs induce CD40-independent immunoglobulin class switching through BLyS and APRIL. Nat Immunol 3, 822-9 (2002).

43. Abu-Rish, E.Y., Amrani, Y. \& Browning, M.J. Toll-like receptor 9 activation induces expression of membrane-bound B-cell activating factor (BAFF) on human B cells and leads to increased proliferation in response to both soluble and membrane-bound BAFF. Rheumatology (Oxford) 52, 1190-201 (2013).

44. Mosak, J. \& Furie, R. Breaking the ice in systemic lupus erythematosus: belimumab, a promising new therapy. Lupus 22, 361-71 (2013).

45. Isenberg, D.A. et al. Efficacy and safety of subcutaneous tabalumab in patients with systemic lupus erythematosus: results from ILLUMINATE-1, a 52-week, phase III, multicentre, randomised, double-blind, placebo-controlled study. Ann Rheum Dis (2015).

46. Merrill, J.T. et al. Efficacy and safety of subcutaneous tabalumab, a monoclonal antibody to B-cell activating factor, in patients with systemic lupus erythematosus: results from ILLUMINATE-2, a 52-week, phase III, multicentre, randomised, double-blind, placebo-controlled study. Ann Rheum Dis (2015).

47. Jacob, C.O. et al. Development of systemic lupus erythematosus in NZM 2328 mice in the absence of any single BAFF receptor. Arthritis Rheum 65, 1043-54 (2013).

48. Zheng, N., Wang, D., Ming, H., Zhang, H. \& Yu, X. BAFF promotes proliferation of human mesangial cells through interaction with BAFF-R. BMC Nephrol 16, 72 (2015).

49. Figgett, W.A. et al. Deleting the BAFF receptor TACI protects against systemic lupus erythematosus without extensive reduction of B cell numbers. J Autoimmun 61, 9-16 (2015).

50. O'Connor, B.P. et al. BCMA is essential for the survival of long-lived bone marrow plasma cells. J Exp Med 199, 91-8 (2004).

51. Vincent, F.B., Morand, E.F., Schneider, P. \& Mackay, F. The BAFF/APRIL system in SLE pathogenesis. Nat Rev Rheumatol 10, 365-73 (2014).

52. Coquery, C.M. \& Erickson, L.D. Regulatory roles of the tumor necrosis factor receptor BCMA. Crit Rev Immunol 32, 287-305 (2012).

53. Jiang, C., Loo, W.M., Greenley, E.J., Tung, K.S. \& Erickson, L.D. B cell maturation antigen deficiency exacerbates lymphoproliferation and autoimmunity in murine lupus. J Immunol 186, 613647 (2011).

54. Jacob, C.O. et al. Differential Development of Systemic Lupus Erythematosus in NZM 2328 Mice Deficient in Discrete Pairs of BAFF Receptors. Arthritis Rheumatol 67, 2523-35 (2015).

55. Huard, B., Schneider, P., Mauri, D., Tschopp, J. \& French, L.E. T cell costimulation by the TNF ligand BAFF. J Immunol 167, 6225-31 (2001). 
56. Ng, L.G. et al. B cell-activating factor belonging to the TNF family (BAFF)-R is the principal BAFF receptor facilitating BAFF costimulation of circulating T and B cells. $J$ Immunol 173, 807-17 (2004).

57. Coquery, C.M. et al. BAFF regulates follicular helper $t$ cells and affects their accumulation and interferon- $\gamma$ production in autoimmunity. Arthritis Rheumatol 67, 773-84 (2015).

58. Goenka, R. et al. Local BLyS production by $\mathrm{T}$ follicular cells mediates retention of high affinity B cells during affinity maturation. $J$ Exp Med 211, 45-56 (2014).

59. Goenka, R., Scholz, J.L., Sindhava, V.J. \& Cancro, M.P. New roles for the BLyS/BAFF family in antigen-experienced B cell niches. Cytokine Growth Factor Rev 25, 107-13 (2014).

60. Scholz, J.L. \& Cancro, M.P. Resolve, revise, and relax: the 3 Rs of B cell repertoire adjustment. Immunol Lett 143, 2-8 (2012).

61. Turner-Stokes, T. et al. The efficacy of repeated treatment with B-cell depletion therapy in systemic lupus erythematosus: an evaluation. Rheumatology (Oxford) 50, 1401-8 (2011).

62. Cambridge, G. et al. The effect of B-cell depletion therapy on serological evidence of B-cell and plasmablast activation in patients with rheumatoid arthritis over multiple cycles of rituximab treatment. J Autoimmun 50, 67-76 (2014).

63. Smilek, D.E., Ehlers, M.R. \& Nepom, G.T. Restoring the balance: immunotherapeutic combinations for autoimmune disease. Dis Model Mech 7, 503-13 (2014).

64. Gong, Q. et al. Importance of cellular microenvironment and circulatory dynamics in B cell immunotherapy. J Immunol 174, 817-26 (2005).

65. Lin, W. et al. Dual B cell immunotherapy is superior to individual anti-CD20 depletion or BAFF blockade in murine models of spontaneous or accelerated lupus. Arthritis Rheumatol 67, 21524 (2015).

66. Wild, J. et al. Neutralization of (NK-cell-derived) B-cell activating factor by Belimumab restores sensitivity of chronic lymphoid leukemia cells to direct and Rituximab-induced NK lysis. Leukemia (2015).

67. https://clinicaltrials.gov/ct2/show/NCT02260934.

68. https://clinicaltrials.gov/ct2/show/NCT02284984.

\section{Author contributions}

Both authors contributed to all aspects of the article: researching data for the article, writing the manuscript, discussions of its content and review or editing of manuscript before submission. 


\section{Figure legends}

Figure 1: BAFF activates and perpetuates interactions between plasmablasts and $\mathbf{T}_{\mathrm{FH}}$ cells in SLE. SLE is characterized by increased proportions of circulating plasmablasts and $\mathrm{T}_{\mathrm{FH}}$ cells. Dendritic cells in patients with SLE produce BAFF, which stimulates plasmablast differentiation. The role of plasmablasts is not limited to the production of pathogenic autoantibodies; they also induce the differentiation of $\mathrm{T}_{\mathrm{FH}}$ cells, via IL-6, which in turn stimulates plasmablast formation, via IL-21. $\mathrm{T}_{\mathrm{FH}}$ cells from lupus-prone mice express both BAFF-R and BCMA, but $\mathrm{T}_{\mathrm{FH}}$ cells from patients with SLE have so far been shown to express only BAFF-R. Thus, the BAFF surge that is characteristic of post-rituximab flares of SLE serves to perpetuate plasmablasts and $\mathrm{T}_{\mathrm{FH}}$ cells, creating a positive feedback loop and worsening antibody-driven inflammation. Furthermore, $\mathrm{T}_{\mathrm{FH}}$ cells in germinal centres can also produce BAFF, thereby promoting the survival of high-affinity germinal centre B cells. Excess BAFF produced by $\mathrm{T}_{\mathrm{FH}}$ cells might provide the necessary survival signals to foster autoreactive B cells in SLE.

Figure 2: The relationship between BAFF, ant-DNA antibodies and B-cell numbers in SLE, before and after rituximab therapy. Active SLE (SLE relapse pre-rituximab therapy) is associated with a modest B-cell lymphopenia and an increased proportion of plasmablasts, compared with the situation in healthy individuals. Those patients who flare after treatment with rituximab with elevated anti-DNA antibodies also have high BAFF levels, often with very low B cell numbers among which autoreactive plasmablasts predominate. This phenomenon can be compounded by repeated rituximab infusions (see also Figure 3). By contrast, patients who remain in remission after B-cell depletion have moderate BAFF levels. Understanding the relationship between BAFF levels, B cell subsets and SLE flare will be crucial for the appropriate selection of therapy in these patients.

\section{Figure 3: The effects of repeated rituximab infusions on anti-dsDNA antibody levels in a} patient with SLE. The patient presented at the age of 26 years with fevers, arthritis and serositis, and was diagnosed with systemic lupus erythematosus (SLE). She was positive for antinuclear antibodies, anti-dsDNA antibodies, antiribonucleoprotein antibodies and anti-Ro antibodies. She failed to respond to conventional treatment with azathioprine, hydroxychloroquine and methotrexate, and went on to develop lupus nephritis. Increasing disease activity led to the commencement of cyclophosphamide and rituximab therapy. Despite several cycles of rituximab over a 5-year period she progressed to end-stage renal 
failure. Her anti-dsDNA antibody levels showed a stepwise increase after almost every rituximab infusion despite low numbers of B cells. B-cell numbers and anti-dsDNA antibody levels are shown, with rituximab treatments indicated by blue arrows. 


\section{Author Biographies}

Michael Ehrenstein

Michael Ehrenstein PhD FRCP is Professor of Experimental Rheumatology at University College London, UK, and Honorary Consultant Rheumatologist at University College London Hospitals. The objectives of his research group are to use biologic therapy as a molecular scalpel to understand the pathogenesis of autoimmune rheumatic diseases, focusing on systemic lupus erythematosus (SLE) and rheumatoid arthritis (RA), both in terms of loss of immune tolerance and ongoing inflammation; development of novel therapies and improvements to existing therapies for patients with SLE and RA; and identification of immune biomarkers that can predict response to treatment in SLE and RA.

\section{Charlotte Wing}

Dr Charlotte Wing MRCP is a Clinical Research Fellow at University College London, UK, where she is undertaking specialist training as a rheumatologist. She completed her medical training at the University of Cambridge, UK and Imperial College School of Medicine, London, UK, graduating with Distinction in 2007. 